International Journal of Pure and Applied Mathematics

Volume 89 No. 3 2013, 343-352

ISSN: $1311-8080$ (printed version); ISSN: 1314-3395 (on-line version)

url: http://www.ijpam.eu

doi: http://dx.doi.org/10.12732/ijpam.v89i3.5

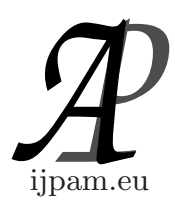

\title{
ON T-FUZZY ESSENTIAL IDEALS OF RINGS
}

\author{
Ujwal Medhi ${ }^{1}$, Helen K. Saikia \\ ${ }^{1}$ Department of Mathematics \\ Girijananda Chowdhury Institute of Management and Technology \\ Guwahati, 781017, INDIA \\ ${ }^{2}$ Department of Mathematics \\ Gauhati University \\ Guwahati, 781014, INDIA
}

\begin{abstract}
In this paper our attempt is to extend the notion of essential fuzzy ideals of rings to T-fuzzy essential ideals. Various properties on T-fuzzy essential ideals are established. Some results are being discussed which establish necessary and sufficient condition between T-fuzzy essential ideal in fuzzy set theory and essential ideal in crisp set theory. Moreover it is proved that if $\mu, \nu$ and $\sigma$ are non-zero T-fuzzy left ideals of $R$ such that $\mu \subseteq \nu \subseteq \sigma$, then $\mu \subseteq \subseteq_{e(T)} \sigma$ if and only if $\mu \subseteq_{e(T)} \nu \subseteq_{e(T)} \sigma$.
\end{abstract}

AMS Subject Classification: $03 \mathrm{E} 72$

Key Words: essential fuzzy ideal, T-fuzzy essential ideal

\section{Introduction}

The notion of fuzzy set was introduced by L.A. Zadeh [13] in 1965. Since then these ideas have been applied to other algebraic structures like groups, rings, modules, topologies and so on. The concept of fuzzy group was introduced by Rosenfeld [12] in 1971. The study of fuzzy sub rings and fuzzy ideals of rings was initiated by W. Liu [7] around 1982. Mukherjee and Sen [10], [11] characterized regular rings in terms of fuzzy ideals of rings. Abou-Zaid [1], Kumar et al [5], [6] and Malik et al [8] have carried out extensive work on fuzzy

Received: $\quad$ May 27, 2013

(c) 2013 Academic Publications, Ltd. url: www.acadpubl.eu 
ideals of rings. U. Medhi et al [9] studied essential fuzzy ideals of rings. The concept of t-norm(i.e. triangular norm) was studied by $\mathrm{D}$ dubois and $\mathrm{H}$ Prade [4]. Dheena and Mohanraaj [3] studied T-fuzzy ideals of rings. In this paper we extend the notion of fuzzy essential ideals of rings to T-fuzzy essential ideals and various properties are being investigated.

\section{Preliminaries}

Throughout this paper $R$ denotes a non-commutative ring with unity unless and otherwise stated.

Definition 2.1. A mapping $T:[0,1] \times[0,1] \rightarrow[0,1]$ is called a triangular norm [T-norm] if and only if it satisfies the following conditions:

T1) $T(x, 1)=T(1, x)=1, \forall x \in[0,1]$.

T2) If $x \geq x^{*}, y \geq y^{*}$ then $T(x, y) \geq T\left(x^{*}, y^{*}\right)$.

T3) $T(x, y)=T(y, x), \forall x, y \in[0,1]$.

T4) $T(x, T(y, z))=T(T(x, y), z), \forall x, y, z \in[0,1]$.

Note. The T-norm minimum (min T-norm) is defined by $T(x, y)=\min (x, y)$. Some other T-norms are $T_{p}(x, y)=x y, T_{n}(x, y)=\max (x+y-1,0)$ and

$$
T_{w}(x, y)= \begin{cases}x, & \text { if } y=1 \\ y, & \text { if } x=1 \\ o, & \text { otherwise }\end{cases}
$$

Every T-norm $T^{*}$ satisfies the inequality:

$$
T_{w}(x, y) \leq T^{*}(x, y) \leq \min (x, y) \text { and } T^{*}(0,0)=0 .
$$

A triangular norm is called an Archimedian t-norm if it satisfies the following conditions:

$\mathrm{T} 5) \mathrm{T}$ is a continuous function (continuity)

T6) $T(x, x)<x, \forall x \in[0,1]$ (subidempotency)

Moreover, if $\mathrm{T}$ also satisfies ( $\mathrm{T} 7$ ), where

T7) $x_{1}<x_{2}, y_{1}<y_{2} \Rightarrow T\left(x_{1}, y_{1}\right)<T\left(x_{2}, y_{2}\right)$ (strict monotnicity)

is called a strict Archimedian t-norm. 
Definition 2.2. A fuzzy subset $\mu$ of $R$ is called a fuzzy left ideal if it satisfies the following properties:

(i) $\mu(x-y) \geq \mu(x) \wedge \mu(y)$, for all $x, y \in R$

(ii) $\mu(x y) \geq \mu(y)$, for all $x, y \in R$

Definition 2.3. A fuzzy subset $\mu$ of $R$ is called a fuzzy right ideal if it satisfies the following properties:

(i) $\mu(x-y) \geq \mu(x) \wedge \mu(y)$, for all $x, y \in R$.

(ii) $\mu(x y) \geq \mu(x)$, for all $x, y \in R$

Definition 2.4. A fuzzy subset $\mu$ of $R$ is called a fuzzy ideal if it satisfies the following properties:

(i) $\mu(x-y) \geq \mu(x) \wedge \mu(y)$, for all $x, y \in R$.

(ii) $\mu(x y) \geq \mu(x) \vee \mu(y)$, for all $x, y \in R$

Definition 2.5. (see [9]) Let $\mu$ be a fuzzy subset of a non-empty set $X$. Then a fuzzy point $x_{t}, x \in X, t \in(0,1]$ is defined as the fuzzy subset $x_{t}$ of $X$ such that $x_{t}(x)=t$, and $x_{t}(y)=0$, for all $y \in X-x$. We write $x_{t} \in \mu$ if and only if $x \in \mu_{t}$.

Definition 2.6. (see [3]) Let $\mu, \lambda$ be two fuzzy subsets of a set $X$. A fuzzy subset $\mu \wedge \lambda$ is defined as $(\mu \wedge \lambda)(x)=T(\mu(x), \lambda(x))$.

Definition 2.7. (see [3]) Let $\mu, \lambda$ be two fuzzy subsets of a set $X$. The product of the fuzzy subsets $\mu$ and $\lambda$ is defined as:

$$
(\mu \lambda)(x)=\sup _{x=y z} T(\mu(y), \lambda(z))
$$

Definition 2.8. (see [3]) A fuzzy subset $\mu$ of a ring $R$ is called T-fuzzy left(right) ideal if:

(i) $\mu(x-y) \geq T(\mu(x), \mu(y))$,

(ii) $\mu(x y) \geq \mu(y)(\mu(x y) \geq \mu(x))$ forall $x, y \in R$.

Notes. (1) If we take T-norm as min-norm T-fuzzy right coincides with fuzzy right ideal.

(2) T-fuzzy ideal is both T-fuzzy right and left ideal. 
Lemma 2.1. (see [3]) Every fuzzy right ideal of a ring $R$ is a $T$-fuzzy right ideal.

Lemma 2.2. (see [3]) If $A$ is a right ideal of a ring $R$, then $\chi_{A}$ is a T-fuzzy right ideal.

Theorem 2.1. (see [3]) If $\mu, \lambda$ are T-fuzzy right ideals of a ring $R$, then $\mu \wedge \lambda$ is a T-fuzzy right ideal of $R$.

Remark 2.1. (see [3]) Every T- fuzzy right ideal need not be a fuzzy ideal by the following example:

Example 1. Consider the ring $R=\{0, a, b, c\}$ with addition and multiplication operations defined as follows:

\begin{tabular}{|l|l|l|l|l|}
\hline+ & 0 & $a$ & $b$ & $c$ \\
\hline 0 & 0 & $a$ & $b$ & $c$ \\
\hline$a$ & $a$ & 0 & $c$ & $b$ \\
\hline$b$ & $b$ & $c$ & 0 & $a$ \\
\hline$c$ & $c$ & $b$ & $a$ & 0 \\
\hline
\end{tabular}

and

\begin{tabular}{|l|l|l|l|l|}
\hline$\cdot$ & 0 & $a$ & $b$ & $c$ \\
\hline 0 & 0 & 0 & 0 & 0 \\
\hline$a$ & 0 & $a$ & $b$ & $c$ \\
\hline$b$ & 0 & 0 & 0 & 0 \\
\hline$c$ & 0 & $a$ & $b$ & $c$ \\
\hline
\end{tabular}

Now we define a fuzzy subset $\mu$ of $R$ such that $\mu(0)=1, \mu(a)=.3, \mu(b)=$ $.5, \mu(c)=.4$

Then $\mu$ is a T-fuzzy ideal of $R$ while it is not a fuzzy ideal of $R$ as

$$
\mu(b+c)=\mu(a)=.3 \ngtr \mu(b) \wedge \mu(c)=.5 \wedge .4 .
$$

Definition 2.9. (see [9]) A non zero fuzzy left ideal $\mu$ of $R$ is called a essential fuzzy left ideal of $R$, denoted by $\mu \subseteq_{e} R$, if for every nonzero fuzzy left ideal $\delta$ of $R$, there exist $x(\neq 0) \in R$ such that $x_{t} \in \mu$ and $x_{t} \in \delta$, for some $t \in(0,1]$.

Definition 2.10. (see [9]) Let $\mu$ and $\sigma$ be two nonzero fuzzy left ideals of $R$ such that $\mu \subseteq \sigma$. Then $\mu$ is called a essential fuzzy left ideal in $\sigma$, denoted by $\mu \subseteq_{e} \sigma$ if for every nonzero fuzzy left ideal $\delta$ of $R$ satisfying $\delta \subseteq \sigma$, there exist $x(\neq 0) \in R$ such that $x_{t} \in \mu$ and $x_{t} \in \delta$, for some $t \in(0,1]$. 


\section{T-Fuzzy Essential Ideal of Rings}

Definition 3.1. A non-zero T-fuzzy left ideal $\mu$ of $R$ is called a T-fuzzy essential left ideal of $R$, denoted by $\mu \subseteq_{e(T)} R$ if for every non-zero T-fuzzy left ideal $\sigma$ of $R, \mu \wedge \sigma \neq \chi_{0}$ i.e there exists $x(\neq 0) \in R$ such that $T[\mu(x), \sigma(x)] \geq t$, for some $t \in(0,1]$.

Example 2. Consider the ring $R=\{0, a, b, c\}$ with addition and multiplication operations defined as follows:

\begin{tabular}{|l|l|l|l|l|}
\hline+ & 0 & $a$ & $b$ & $c$ \\
\hline 0 & 0 & $a$ & $b$ & $c$ \\
\hline$a$ & $a$ & 0 & $c$ & $b$ \\
\hline$b$ & $b$ & $c$ & 0 & $a$ \\
\hline$c$ & $c$ & $b$ & $a$ & 0 \\
\hline
\end{tabular}

and

\begin{tabular}{|l|l|l|l|l|}
\hline$\cdot$ & 0 & $a$ & $b$ & $c$ \\
\hline 0 & 0 & 0 & 0 & 0 \\
\hline$a$ & 0 & $a$ & $b$ & $c$ \\
\hline$b$ & 0 & 0 & 0 & 0 \\
\hline$c$ & 0 & $a$ & $b$ & $c$ \\
\hline
\end{tabular}

Now we define a fuzzy subset $\mu$ of $R$ such that $\mu(0)=1, \mu(a)=.4, \mu(b)=$ $.6, \mu(c)=.5$.

Suppose $\sigma$ is a non zero T-fuzzy left ideal of $R$. Then there exists $x(\neq 0) i n R$ such that $\sigma(x)=t(\neq 0)$, where $t \in(0,1]$. Suppose $\sigma(a) \neq 0$.

Now we consider the product norm $T_{p}$ defined by $T_{p}(s, t)=s t$, where $s, t \in$ $[0,1]$.

Then clearly $T_{p}(\mu(a), \sigma(a))=\mu(a) \sigma(a) \neq 0$. Hence $\mu$ is a T-fuzzy essential left ideal of $R$.

But $\mu$ is not a T-fuzzy essential under the norm $T=T_{w}$, where $T_{w}$ is defined as follows:

$$
T_{w}(s, t)=\max \{s+t-1,0\}, \text { for the non zero T-fuzzy ideal } \sigma,
$$

where $\sigma(0)=1, \sigma(a)=.3, \sigma(b)=.5, \sigma(c)=.4$ is such that there exists no non zero x satisfying $T_{w}(\mu(x), \sigma(x)) \neq 0$.

Note. For the next results except theorems 3.2,3.4,3.5 of this section we consider $\mathrm{T}$ as an Archimedian t-norm. 
Theorem 3.1. Let $\mu$ be a T-fuzzy essential left ideal of $R$. Then $\mu$ is a T-fuzzy essential left ideal of $R$ if and only if $\mu_{t}$ is an essential left ideal of $R$, for some $t \in(0,1]$.

Proof. Let $\mu$ be a T-fuzzy essential left ideal of $R$. Let $A$ be any non-zero left ideal of $R$. Then $\chi_{A}$ is a non-zero T-fuzzy left ideal of $R$. Since $\mu \subseteq_{e(T)} R$, so we have $\mu \wedge \chi_{A} \neq \chi_{0}$, there exists $x(\neq 0) \in R$ such that $T\left[\mu(x), \chi_{A}(x)\right] \neq 0$. Thus, there exists $x(\neq 0) \in R$ such that $T\left[\mu(x), \chi_{A}(x)\right] \geq t$ for some $t \in(0,1]$. Now, $\chi_{A}(x), \mu(x) \geq \min \left[\chi_{A}(x), \mu(x)\right] \geq T\left[\chi_{A}(x), \mu(x)\right] \geq t$. This implies $\mu(x) \geq t$ and $\chi_{A}(x) \geq t, t \neq 0$ and thus $x \in \mu_{t} \cap A$. Hence $\mu_{t}$ is an essential left ideal of $R$.

Conversely, let $\mu_{t} \subseteq_{e} R$. Then, for some $t \in(0,1], \mu_{t}$ is non-zero for which $\mu$ is non-zero. Let $\sigma$ be any non-zero T-fuzzy left ideal of $R$. Then there exists $x(\neq 0) \in R$ such that $\sigma(x)=s(\neq 0)$. As $\mu_{t}$ is an essential left ideal of $R$, there exists $y(\neq 0) \in R$ such that $y \in \mu_{t} \cap \sigma_{s}$. This implies $\mu(y) \geq t$, $\sigma(y) \geq s$ and therefore $\mu(y) \geq t_{0}, \sigma(y) \geq t_{0}$ where $t_{0}=\min \{s, t\}$. Also, $(\mu \wedge \sigma)(y)=T[\mu(y), \sigma(y)] \geq T(s, t)>T(0,0)=0$. Thus for $y(\neq 0) \in R$ we get $(\mu \wedge \sigma)(y) \neq 0$ i.e. $\mu \wedge \sigma \neq \chi_{0}$. Hence $\mu$ is a T-fuzzy essential left ideal of $R$.

Theorem 3.2. A left ideal $A$ of $R$ is essential in $R$ if and only if $\chi_{A}$ is a $T$-fuzzy essential left ideal of $R$.

Proof. Let $A \subseteq e R$. Then $\chi_{A}$ is a non-zero T-fuzzy left ideal of $R$. Let $\mu$ be any non-zero T-fuzzy left ideal of $R$. Then $\mu_{t}$ is a non-zero left ideal of $R$ where $t \in(0,1]$. Then there exists $x(\neq 0) \in R$ such that $x \in \mu_{t} \cap A$ i.e. $\mu(x) \geq t$ and $\chi_{A}(x)=1$. Now, $\left(\chi_{A} \wedge \mu\right)(x)=T\left[\mu(x), \chi_{A}(x)\right]=T[\mu(x), 1]=\mu(x) \geq t>0$. Hence $\chi_{A} \subseteq_{e(T)} R$.

Conversely, let $A$ be a left ideal of $R$ such that $\chi_{A}$ is T-fuzzy essential in $R$. Let $B$ be any non-zero left ideal of $R$. Then $\chi_{B}$ is a non-zero T-fuzzy left ideal of $R$. This implies there exists $x(\neq 0) \in R$ such that $T\left[\chi_{A}(x), \chi_{B}(x)\right] \geq t$ for some $t \in(0,1]$. Now $\chi_{A}(x), \chi_{B}(x) \geq \min \left[\chi_{A}(x), \chi_{B}(x)\right] \geq T\left[\chi_{A}(x), \chi_{B}(x)\right] \geq t$. Thus we get, $\chi_{A}(x) \geq t$ and $\chi_{B}(x) \geq t$. Thus we have, $\chi_{A}(x)=1$ and $\chi_{B}(x)=1$. This implies $x \in A$ and $x \in B$. Hence $A$ is an essential left ideal of $R$.

Lemma 3.1. A T-fuzzy left ideal $\mu$ of $R$ is a T-fuzzy essential left ideal of $R$, if and only if $\mu$ is a $T$-fuzzy essential left ideal of $\chi_{R}$. left ideal of $R$.

Proof. Let $\mu \subseteq_{e(T)} R$. Let $\sigma \subseteq \chi_{R}$ be any non-zero T-fuzzy left ideal of $R$. Since $\mu \subseteq_{e(T)} R$, so we have $\mu \wedge \sigma \neq \chi_{0}$. Therefore $\mu \subseteq e(T) \chi_{R}$. 
Conversely, let $\mu \subseteq_{e(T)} \chi_{R}$. Let $\sigma$ be any non-zero T-fuzzy left ideal of $R$. Then clearly $\sigma \subseteq \chi_{R}$. Also $\mu \subseteq_{e(T)} \chi_{R}$ and therefore $\mu \wedge \sigma \neq \chi_{0}$. Hence $\mu \subseteq_{e(T)} R$.

Definition 3.2. Let $\mu$ and $\sigma$ be two non-zero T-fuzzy left ideals of $R$ such that $\mu \subseteq \sigma$. Then $\mu$ is called a T-fuzzy essential left ideal in $\sigma$, denoted by $\mu \subseteq_{e(T)} \sigma$, if for every non-zero T-fuzzy left ideal $\theta$ of $R$ satisfying $\delta \subseteq \sigma$, $\mu \wedge \delta \neq \chi_{0}$ i.e. there exists $x(\neq 0) \in R$ such that $T[\mu(x), \delta(x)] \geq t$, for some $t \in(0,1]$.

Theorem 3.3. If $A$ and $B$ are two non-zero left ideals of $R$, then $A$ is an essential left ideal of $B$ if and only if $\chi_{A}$ is a T-fuzzy essential left ideal in $\chi_{B}$.

Proof. Let $A \subseteq e B$. Then $A \subseteq B$ and therefore $\chi_{A} \subseteq \chi_{B}$. Let $\sigma$ be any non-zero T-fuzzy ideal with $\sigma \subseteq \chi_{B}$. Then $\sigma_{t}$ is a non-zero left ideal and $\sigma_{t} \subseteq B$, for some $t \in(0,1]$. This implies there exists $x(\neq 0) \in R$ such that $x \in \sigma_{t} \cap A$. Therefore $\sigma(x) \geq t$ and $\chi_{A}(x)=1$. Now $\left.T\left[\sigma(x), \chi_{A}(x)\right)\right]=$ $T[\sigma(x), 1)]=\sigma(x) \geq t$. Thus we have, $\sigma \wedge \chi_{A} \neq \chi_{0}$. Hence $\chi_{A} \subseteq_{e(T)} \chi_{B}$.

Conversely, let $\chi_{A} \subseteq \subseteq_{e(T)} \chi_{B}$. Then $A \subseteq B$. Let $C$ be a non-zero left ideal of $R$ with $C \subseteq B$. Then $\chi_{C}$ is non-zero and $\chi_{C} \subseteq \chi_{B}$. Since $\chi_{A} \subseteq e(T)$ $\chi_{B}$, we get $\chi_{A} \wedge \chi_{C} \neq \chi_{0}$. This implies there exists $x(\neq 0) \in R$ such that $T\left[\chi_{A}(x), \chi_{C}(x)\right] \geq t$ for some $t \in(0,1]$.

$$
\text { Now } \begin{aligned}
\chi_{A}(x), \chi_{C}(x) & \geq \min \left[\chi_{A}(x), \chi_{C}(x)\right] \\
& \geq T\left[\chi_{A}(x), \chi_{C}(x)\right] \\
& \geq t
\end{aligned}
$$

Thus $\chi_{A}(x) \geq t$ and $\chi_{C}(x) \geq t$. Thus $x \in A$ and $x \in C$. Hence $A \subseteq_{e} B$.

Theorem 3.4. Let $\mu$ and $\sigma$ be two non-zero T-fuzzy left ideals of $R$ such that $\mu \subseteq \sigma$. Then $\mu \subseteq_{e(T)} \sigma$ if and only if $\mu_{t} \subseteq_{e} \sigma_{t}$, for some $t \in(0,1]$.

Proof. Let $\mu \subseteq_{e(T)} \sigma$. Since $\mu \subseteq \sigma$, we have $\mu_{t} \subseteq \sigma_{t}$, for some $t \in(0,1]$. Let $A$ be any non-zero left ideal of $\sigma_{t}$.

A fuzzy subset $\delta$ of $R$ is defined such that

$$
\delta(x)= \begin{cases}\sigma(x), & \text { if } x \in A \\ 0, & \text { if } x \notin A .\end{cases}
$$

Then $\delta$ is a non-zero T-fuzzy left ideal of $R$ such that $\delta \subseteq \sigma$. Thus there exists $x(\neq 0) \in R$ such that $T[\mu(x), \delta(x)] \geq t$ for some $t \in(0,1]$. 
Now $\mu(x), \delta(x) \geq \min [\mu(x), \delta(x)] \geq T[\mu(x), \delta(x)] \geq t$.

Thus $\exists x(\neq 0) \in R$ such that $x \in \mu_{t} \cap \delta_{t}$. Hence $\mu_{t} \subseteq_{e} \sigma_{t}$.

Conversely, let $\mu_{t} \subseteq_{e} \sigma_{t}$, for some $t \in(0,1]$. Let $\delta$ be a non-zero T-fuzzy left ideal of $R$ with $\delta \subseteq \sigma$. Then $\delta_{t}$ is a non-zero left ideal of $R$ with $\delta_{t} \subseteq \sigma_{t}$, for all $t \in(0,1]$. Since $\mu_{t} \subseteq_{e} \sigma_{t}$, for some $t \in(0,1]$. So, there exists $x(\neq 0) \in R$ such that $x \in \mu_{t} \cap \delta_{t}$. This implies $\mu(x) \geq t$ and $\delta(x) \geq t$. Now, $(\mu \wedge \delta)(x)=$ $T[\mu(x), \delta(x)] \geq T[t, t)]>T[0,0]=0$ [since $\mathrm{T}$ satisfies Archeamediam property ]. Thus we have, $\mu \wedge \delta \neq \chi_{0}$. Hence $\mu \subseteq_{e(T)} \sigma$.

Lemma 3.2. (see [3]) Let $T$ be a t-norm. Then $T(T(a, b), T(c, d))=$ $T(T(a, c), T(b, d))$, for all $a, b, c, d \in[0,1]$.

Theorem 3.5. Let $\mu$ and $\sigma$ be two non-zero T-fuzzy left ideals of $R$ such that $\mu$ is a T-fuzzy essential left ideal in $\sigma$. Then for any T-fuzzy left ideal $\delta$ of $R, \mu \wedge \delta \subseteq_{e(T)} \sigma \wedge \delta$

Proof. Since $\mu \subseteq \sigma$, so $\mu \wedge \delta \subseteq \sigma \wedge \delta$. Let $\eta$ be any non-zero T-fuzzy left ideal of $R$ such that $\eta \subseteq \sigma \wedge \delta$. Then $\eta$ is a non-zero T-fuzzy left ideal of $R$ such that $\eta \subseteq \sigma$ and $\eta \subseteq \delta$. This implies $\exists x(\neq 0) \in R$ such that $T[\mu(x), \eta(x)] \geq t$.

Now $\mu(x), \eta(x) \geq \min [\mu(x), \eta(x)] \geq T[\mu(x), \eta(x)] \geq t$.

Also $\delta(x) \geq \eta(x)$, for all $x \in R$ this implies $\delta(x) \geq \eta(x) \geq t$, for all $x \in R$.

Therefore

$$
\begin{aligned}
{[(\mu \wedge \delta) \wedge \eta](x) } & =T[(\mu \wedge \delta)(x), \eta(x)] \\
& =T[T(\mu(x), \delta(x)), \eta(x)] \\
& =T[\mu(x), T(\delta(x), \eta(x))] \\
& =T[\mu(x), T(\eta(x), \delta(x))] \\
& =T[T(\mu(x), \eta(x)), \delta(x)] \\
& \geq T[T(\mu(x), \eta(x)), t] \\
& \geq T(t, t)>T(0,0)=0,
\end{aligned}
$$

since $T$ satisfies Archeamediam property.

Thus we get $(\mu \wedge \delta) \wedge \eta \neq \chi_{0}$. Hence $\mu \wedge \delta \subseteq_{e(T)} \sigma \wedge \delta$.

Theorem 3.6. Let $\mu, \nu$ and $\sigma$ be non-zero T-fuzzy left ideals of $R$ such that $\mu \subseteq \nu \subseteq \sigma$. Then $\mu \subseteq_{e(T)} \sigma$ if and only if $\mu \subseteq_{e(T)} \nu \subseteq_{e(T)} \sigma$.

Proof. Let $\mu \subseteq_{e(T)} \sigma$. Let $\delta$ be a non-zero T-fuzzy left ideal of $\nu$. Then $\delta$ is also a non-zero T-fuzzy left ideal of $\sigma$. Thus there exists $x(\neq 0) \in R$ such that $T[\mu(x), \delta(x)] \geq t$, for some $t \in(0,1]$. Consequently $\mu \subseteq_{e(T)} \nu$. Again, let $\xi$ be 
any non-zero T-fuzzy left ideal of $\sigma$. Since $\mu \subseteq_{e(T)} \sigma$, so there exists $x(\neq 0) \in R$ such that $T[\mu(x), \delta(x)] \geq t$, for some $t \in(0,1]$ and this implies $\nu \subseteq_{e(T)} \sigma$.

Conversely, let $\mu \subseteq_{e(T)} \nu \subseteq_{e(T)} \sigma$. Let $\eta$ be a non-zero T-fuzzy left ideal of $\sigma$. Since $\nu \subseteq_{e(T)} \sigma$, so there exists $x(\neq 0) \in R$ such that $T[\nu(x), \eta(x)] \geq t$, for some $t \in(0,1]$. Also $\eta \wedge \nu$ is a non-zero T-fuzzy left ideal of $\nu$ and $\mu \subseteq_{e(T)} \nu$. This implies there exists $y(\neq 0) \in R$ such that $T[\mu(y),(\nu \wedge \eta)(y)] \geq s$, for some $s \in(0,1]$.

Now $\mu(y),(\nu \wedge \eta)(y) \geq \min [\mu(y),(\nu \wedge \eta)(y)] \geq T[\mu(y),(\nu \wedge \eta)(y)] \geq s$.

Also $\nu(y), \eta(y) \geq \min [\nu(y), \eta(y)] \geq T[\nu(y), \eta(y)] \geq s$.

Thus $(\mu \wedge \eta)(y)=T[\mu(y), \eta(y)] \geq T(s, s)>T(0,0)=0$. This implies there exists $y(\neq 0) \in R$ such that $T[\mu(y), \eta(y)] \geq s$, for some $s \in(0,1]$.

Thus we have $\mu \wedge \eta \neq \chi_{0}$. Hence $\mu \subseteq_{e(T)} \sigma$.

\section{References}

[1] Abou-Zaid S. On fuzzy ideals and fuzzy quotient rings of a ring. Fuzzy sets and systems, 59(1993), 205-210.

[2] Chatters A.W and Hazarnavis C.R. Rings with chain condition. Pitman Advanced Publishing program, (1980).

[3] Dheena P. and Mohanraaj G., T-fuzzy Ideals in Rings, International Journal of Computational Cognition, Volume 9, No.2(june 2011), 98-101.

[4] Dubois D. and Prade H., Theory and Applications,Fuzzy sets and systems, Academic Press, New York (1978).

[5] Kumar R. Fuzzy ideals and fuzzy semi-prime ideals. Some ring theoretic analogues, Information Sci. 46(1992), 43-52.

[6] Kumar R. Certain fuzzy ideals of rings redefined. Fuzzy sets and systems, 46(1992), 251-260.

[7] Liu. W Fuzzy invariant subgroups and fuzzy ideals. Fuzzy sets and systems, 8(1982), 133-139.

[8] Malik D.S. Fuzzy ideals of artinian rings. Fuzzy sets and systems, 46(1990), 111-115.

[9] Medhi.U, Rajkhowa. K. K, Barthakur. L. K. and Saikia H.K. On fuzzy essential ideals of rings. Advances in fuzzy sets and systems, Vol.3(No.3)(2008), 287-299. 
[10] Mukharjee T.K. and Sen M.K. Prime fuzzy ideals in rings. Fuzzy sets and systems, 32(1989), 337-341.

[11] Mukharjee T. K. and Sen M. K. Rings with chain conditions Fuzzy sets and systems, 39(1991), 117-123.

[12] Rosenfeld A. Fuzzy groups. J. Math. anal. Appl. 35(1971), 512-517.

[13] Zadeh L.A. Fuzzy sets. Information and Control, 8(1965), 338-353. 